\title{
Olelo Wehewehe i ka Mea Heluhelu
}

He mau manao keia e hapai aku nei i mua o ka mea heluhelu no na ike i hoike ia i loko o keia puke. He mau manao hoakaka wale no keia i ka mea heluhelu i wahi e ike ai i ke kumu i koho ia ai keia mau mahele o lalo nei, no ka pela ana, na hua olelo i hoohio ia, na hua olelo hou, na hua olelo Niihau, na kaha apo, a me ke kaha puanai.

\section{Ka Pela Ana}

Ua koho au e hahai i ka pela ana o na hua olelo i loko o keia puke e like me ka mea i kakau ia ma ka puke wehewehe olelo a Pukui laua o Elbert. O kekahi kumu no ia koho ana, i mea e kau like ai ke kakau ana ma ka olelo kanaka. O ka pela ana a ka poe Niihau, okoa iki ae mai ko waho nei poe. I kekahi manawa, ua hoohui ia elua hua olelo a i hookahi hua olelo a he ike ia no ia ano pela ana ma na nupepa kahiko kekahi. Oiai, aole no kekahi Niihau i kakau mua i kekahi puke ma ka olelo Niihau, eia nae, ma loko wale no o ke kakau ana i na leka na ka ohana i ike ia ai kela ano pela ana i na hua olelo. I wahi e nani ai ka olelo o keia puke i ke anaina o waho nei, ua hooholo ia e pela e like me ke ano o ke pela ana o keia wa.

\section{Na Hua Olelo Hio}

O ka mea hoi e ike ia ai ka okoa ma waena o ka olelo kanaka a me ka olelo haole, ua hoohio ia na hua olelo i pela ia ma ka haole. A o kekahi o na hua olelo i hoohio ia, he hua olelo no ka poo manao o kekahi puke a i ole i kekahi hua olelo me ka manao ano nui i hoohana ia ma kela me keia mokuna o ka puke.

\section{Na Hua Olelo Hou}

Nui na hua olelo hou i hoohana ia i loko o keia puke. No ka nui o na hua olelo i hoopuka ia i ka waha o na kanaka Niihau, pela no i komo ai i loko o keia puke. 


\section{Na Hua Olelo Niihau}

O na hua olelo Niihau i paa ma keia puke, he mau olelo wale no i hoopuka ia mai ka waha mai o ka poe Niihau. O lakou wale no ka poe i ninaninau ia no keia puke no na hana a lakou i lawelawe ai no ka pono o ke ola kino a me ke ola uhane. $\mathrm{O}$ kekahi mahele nui i ninaninau ia, ko lakou ike, ko lakou noeau, na olelo ao, na manao waiwai i loko o ko lakou ola, ka lakou hana, a me ka noho ana. Ua malama ia na kukakamailio ana ma ka olelo Niihau wale no. E like me ka mea i hoopuka ia mai ka waha mai o ka poe Niihau, pela no i kakau ia ai ma keia puke. He mea waiwai ka ike ana o ka mea heluhelu i ke ano o ka olelo Niihau.

\section{Na Kaha Apo}

O na kaha apo, ua hoohana wale ia no no ka hoike ana i ka inoa o ke kanaka nana ia olelo a me ka makahiki i pai ia ai ka puke.

\section{Na Kaha Puanai}

O na manao i ohiohi ia mai kekahi puke mai, ua hoohana ia ke kaha puanai no ka hoike ana i ka olelo oiaio a ia kanaka. Pela pu na olelo i hoopuka ia i ka waha o ka poe Niihau, ua hoohana ia ka puanai kekahi. E like hoi me ke ano o ke kakau ana a ka poe kakau moolelo ma o ka hoohana ana i ke kaha puanai i mea e hoike aku ai i ka mea heluhelu, he olelo ia mai kekahi kanaka mai. 
MAI PUKAIKI KULA MANIANIA A PUUWAI ALOHA O KA OHANA 
